\title{
Factors Associated with Prompt Recovery among Hospitalized Patients with Coronavirus Disease 2019
}

\author{
Pamela $\mathrm{Ny}^{1}$, Corey Kelsom ${ }^{1}$, Amanda Chron ${ }^{2}$, Mimi Lou ${ }^{2}$, Paul Nieberg ${ }^{1}$, Kimberly \\ Shriner ${ }^{1}$, Holly Huse ${ }^{1}$, and Annie Wong-Beringer ${ }^{1}$ \\ ${ }^{1}$ Huntington Memorial Hospital \\ ${ }^{2}$ University of Southern California
}

February 28, 2021

\begin{abstract}
Background: Patients who survived hospitalization for COVID-19 experienced varying durations of illness but the factors associated with prompt recovery are unknown. This study identifies factors differentiating hospitalized patients who recovered promptly vs. survived a prolonged course of illness due to COVID-19. Methods: This was a retrospective study from March-August 2020 of hospitalized adults with COVID-19 which were grouped based on time to recovery: short ([?] 3 days), intermediate (4-10 days), and prolonged (>10 days). Recovery was defined as resolution of fever, tachypnea, hypotension, extubation and return of mental status at baseline. Multivariate analysis was used to evaluate factors associated with prompt recovery. Results: Among 508 patients hospitalized for COVID-19, 401 (79\%) survived. Of those, prompt recovery (within 3 days) was achieved in $43 \%$ (174/401) whereas $23 \%$ (92/401) recovered after a prolonged period of $>10$ days. Overall, median age was 64 y with $73 \%$ admitted from home and $25 \%$ from a skilled nursing facility. Predictors for prompt recovery upon admission included female sex $(\mathrm{OR}, 1.8 ; 95 \% \mathrm{CI}, 1.1-2.7 ; \mathrm{p}=0.01)$, no fever $(\mathrm{OR}, 1.6 ; 95 \% \mathrm{CI}, 1.1-2.6 ; \mathrm{p}=0.03)$, longer time from symptom onset to hospitalization (OR, 1.1; 95\% CI, 1.0-1.1; p = 0.001), no supplemental oxygen (OR, 1.9; 95\% CI, 1.2-3.0; p $=0.004)$, no direct ICU admission (OR, 41.7; 95\% CI, 2.4-740.4; $\mathrm{p}=0.01)$ and absence of bacterial co-infections (OR, 2.5; 95\% $\mathrm{CI}, 1.5-4.0, \mathrm{p}=0.0003)$. Conclusions: Our study provides relevant data that could help clinicians triage competing resources in health systems that are challenged by the ebb and flow of COVID-19 cases by identifying clinical features of COVID-19 patients who may require less intensive management including avoidance of unnecessary antibacterial therapy.
\end{abstract}

Factors Associated with Prompt Recovery among Hospitalized Patients with Coronavirus Disease 2019

Pamela Ny, PharmD ${ }^{1}$; Corey Kelsom, MS, PharmD ${ }^{1,2}$; Amanda Chron, $\mathrm{MS}^{2}$; Mimi Lou, $\mathrm{MS}^{2}$, Paul Nieberg, $\mathrm{MD}^{1}$; Kimberly Shriner, $\mathrm{MD}^{1}$; Holly Huse, $\mathrm{PhD}^{1}$; Annie Wong-Beringer, $\mathrm{PharmD}^{1,2}$

${ }^{1}$ Huntington Hospital, Pasadena, CA, USA

${ }^{2}$ University of Southern California, Los Angeles, CA, USA

Corresponding Author:

Annie Wong-Beringer, PharmD

University of Southern California School of Pharmacy

1985 Zonal Avenue, Los Angeles, CA 90089-9121

(323) 442-1356

Email: anniew@usc.edu 
Short running title (max 40 characters): Favorable risk factors in COVID-19

Key words: COVID-19, prompt recovery, antimicrobial stewardship

\section{Acknowledgments}

This work was supported by grants UL1TR001855 and UL1TR000130 from the National Center for Advancing Translational Science (NCATS) of the U.S. National Institutes of Health. The content is solely the responsibility of the authors and does not necessarily represent the official views of the National Institutes of Health.

\section{Abstract}

Background: Patients who survived hospitalization for COVID-19 experienced varying durations of illness but the factors associated with prompt recovery are unknown. This study identifies factors differentiating hospitalized patients who recovered promptly vs. survived a prolonged course of illness due to COVID-19.

Methods: This was a retrospective study from March-August 2020 of hospitalized adults with COVID-19 which were grouped based on time to recovery: short ([?] 3 days), intermediate (4-10 days), and prolonged (>10 days). Recovery was defined as resolution of fever, tachypnea, hypotension, extubation and return of mental status at baseline. Multivariate analysis was used to evaluate factors associated with prompt recovery.

Results: Among 508 patients hospitalized for COVID-19, 401 (79\%) survived. Of those, prompt recovery (within 3 days) was achieved in $43 \%(174 / 401)$ whereas $23 \%(92 / 401)$ recovered after a prolonged period of $>10$ days. Overall, median age was 64 y with $73 \%$ admitted from home and $25 \%$ from a skilled nursing facility. Predictors for prompt recovery upon admission included female sex (OR, 1.8; 95\% CI, 1.1-2.7; $p=$ 0.01 ), no fever (OR, 1.6; 95\% CI, 1.1-2.6; $p=0.03$ ), longer time from symptom onset to hospitalization (OR, $1.1 ; 95 \%$ CI, 1.0-1.1; $p=0.001$ ), no supplemental oxygen (OR, 1.9; 95\% CI, 1.2-3.0; $p=0.004$ ), no direct ICU admission (OR, 41.7; 95\% CI, 2.4-740.4; $p=0.01)$ and absence of bacterial co-infections (OR, 2.5; $95 \%$ CI, $1.5-4.0, p=0.0003)$.

Conclusions: Our study provides relevant data that could help clinicians triage competing resources in health systems that are challenged by the ebb and flow of COVID-19 cases by identifying clinical features of COVID-19 patients who may require less intensive management including avoidance of unnecessary antibacterial therapy.

\section{What is already known about this subject?}

Patients who survived hospitalization for COVID-19 experienced varying durations of illness but the factors associated with prompt recovery are unknown

\section{What does this study contribute to the literature?}

Our study provides relevant data that could help clinicians triage competing resources in health systems that are challenged by the ebb and flow of COVID-19 cases by identifying clinical features of COVID-19 patients who may require less intensive management including avoidance of unnecessary antibacterial therapy.

\section{Introduction}

The novel coronavirus, severe acute respiratory syndrome coronavirus 2 (SARS-CoV-2), caused an initial outbreak in Wuhan, China in December 2019 and has spread resulting in a global pandemic with significant mortality and strain on healthcare operations. ${ }^{1-3}$ To date, most of the published work focuses on the epidemiological, clinical, laboratory and radiological characteristics of patients infected with SARS-CoV-2 along with risk factors associated with mortality. Less is known about the varying durations of illness among those who survived hospitalization for COVID-19. With many health systems challenged by the demands from the ebb and flow of COVID-19 cases nationwide and globally, our study aims to identify features associated with prompt recovery to help clinicians triage management of patients hospitalized for COVID-19. 


\section{Methods}

This retrospective cohort study was conducted on adult patients (age [?]18 years) who survived hospitalization for symptomatic COVID-19 confirmed by positive polymerase chain reaction (PCR) result for SARS-CoV-2 from March to August 2020 at a 625-bed community teaching hospital in Los Angeles. The study was approved by the hospital's Institutional Review Board; informed consent was waived.

Medical charts were reviewed to obtain demographic, laboratory, and clinical information, and details of antiviral and antibacterial therapy. All data were recorded on a structured data collection form and managed using REDCap electronic data capture tools hosted at the University of Southern California. ${ }^{4}$ Sepsis-3 criteria were used to define sepsis and septic shock. ${ }^{5}$ Outcome measures included development of acute kidney injury (AKI) and acute respiratory distress syndrome (ARDS) and hospital length of stay (LOS).

\section{Data analysis}

Patients were grouped based on short ([?] 3 days), intermediate (4-10 days), prolonged ( $>10$ days) time to recovery defined by achievement of clinical stability (TTS): resolution of fever $<38^{\circ} \mathrm{C}$, respiratory rate $<$ 22 breaths/min, systolic blood pressure $>100 \mathrm{mmHg}$, extubated, and return of mental status to baseline. Study groups were compared on demographic and clinical characteristics, pharmacologic management and outcomes. Descriptive analyses were performed using Kruskal-Wallis and chi-square test where appropriate. Predictors for prompt recovery after controlling for significant variables were analyzed by univariate and multivariable logistic regression. A $p$ value $<0.05$ was considered significant. Statistical analyses were performed using GraphPad Prism v6.07 (San Diego, CA, USA) or SAS version 9.4 (SAS Institute, Cary, NC).

\section{Results}

Survival rate was $79 \%$ (401/508) among patients hospitalized for COVID-19 during the study period. Overall, median age of COVID-19 survivors was 64 years; majority (73\%) were admitted from home with $25 \%$ from a skilled nursing facility (SNF). The overall median TTS was 4 days (IQR: 2-10) for survivors, with $43 \%$ $(\mathrm{n}=174)$ within 3 days, $34 \%(\mathrm{n}=135)$ between $4-10$ days, and $23 \%(\mathrm{n}=92)$ after a prolonged period exceeding 10 days. More females achieved prompt vs. prolonged time to recovery ( $51 \%$ vs. $37 \%$ ) (Table 1 ).

At time of admission, significantly less patients in the short TTS group experienced fevers (47\% vs. $58 \%$ vs. $63 \%, p=0.0214)$, required supplemental oxygen ( $55 \%$ vs. $73 \%$ vs. $77 \%, p<0.0001)$, while none had septic shock (vs. $4 \%$ vs. $16 \%, \mathrm{p}<0.0001$ ), required direct ICU admission (vs. $7 \%$ vs. $25 \%, p<0.0001$ ) or mechanical ventilation (vs. $2 \%$ vs. $16 \%, p<0.0001$ ) compared to the intermediate and prolonged groups, respectively (Table 1 ). Therapy directed against COVID-19 was less frequently prescribed to patients with short compared to intermediate and prolonged TTS: hydroxychloroquine \pm azithromycin $(13 \%$ vs. $32 \%$ vs. $37 \%, p<0.0001)$, remdesivir ( $14 \%$ vs. $29 \%$ vs. $48 \%, p<0.0001)$, tocilizumab ( $0.6 \%$ vs. $0.7 \%$ vs. $12 \%, p<$ $0.0001)$, corticosteroids ( $34 \%$ vs. $31 \%$ vs. $58 \%, p<0.0001$ ), and convalescent plasma ( $10 \%$ vs. $24 \%$ vs. $40 \%$, $p<0.0001)$.

Patients with prompt recovery were less likely to have secondary bacterial infections at time of admission (presumed $13 \%$ vs. $24 \%$ vs. $39 \%, p=0.0001$; culture-positive $11 \%$ vs. vs. $21 \%$ vs. $28 \%, p=0.0013$ ), especially in the respiratory site $(0 \%[0 / 19]$ vs. $10 \%[3 / 29]$ vs. $46 \%[12 / 26], p=0.0002)$ compared to intermediate and prolonged TTS groups, respectively. Nonetheless, $83 \%$ of the patients in the short TTS group who did not have secondary bacterial infections were prescribed broad-spectrum antibacterial therapy for a median duration of 4 days. A notable proportion of the prolonged TTS cohort were co-infected with multidrug-resistant pathogens such as Pseudomonas aeruginosa (15\%) and carbapenem-resistant organisms (4\%) compared to none in the short TTS group; patients who resided in a SNF prior to admission accounted for $56 \%(5 / 9)$ of those with Pseudomonas aeruginosa and all three cases involving carbapenem-resistant organisms. Interestingly, co-infection with ESBL-producing isolates (mostly urine) occurred in $4 \%$ of patients overall, irrespective of TTS. Compared to the intermediate and prolonged TTS cohorts, less patients with a short TTS developed AKI (10\% vs. $17 \%$ vs. $34 \%, p<0.0001$ ) and none developed ARDS (vs. $1 \%$ vs. $17 \%, p<$ 0.0001) (Table 1 ). Ultimately, those who recovered fastest experienced a shorter hospital stay (median 5 
vs. 9 vs. 22 days, $p<0.0001$ ) (Table 1 ).

By multivariable logistic regression analysis, independent predictors for prompt recovery from COVID-19 were female sex, absence of fever, longer time from symptom onset to hospitalization, no direct ICU admission, not requiring supplemental oxygen upon presentation and absence of presumed or documented co/secondary bacterial infections (Table 2 ).

\section{Discussion}

Patients who survived hospitalization with COVID-19 experienced varying time to achieve clinical stability before hospital discharge. While much of the focus has been on the severity of COVID-19 among those who required hospitalization, less is known regarding the characteristics of patients who recovered promptly. Clinical features associated with a favorable hospital course could help clinicians triage competing resources in the care of patients especially at institutions challenged by the demands from the ebb and flow in COVID19 cases.

Our findings indicate that female sex, longer time from symptom onset to hospitalization, less severe presentation upon admission (fevers, septic shock, ICU, supplemental oxygen), and absence of bacterial co-infection are factors favoring prompt recovery. We observed a similar prevalence of COVID-19 requiring hospitalization between male and female sex in our study. Although literature has shown a correlation between men with COVID-19 and worse outcomes including death, our data is the first to show an association between female sex and shortened recovery time. ${ }^{6,7}$ Our data is also the first to show that an extended time from symptom onset to hospital admission is a predictor for prompt recovery.

We found that significantly less patients with prompt recovery had bacterial co-infections especially concurrent pneumonia compared to those in the prolonged TTS group. Nonetheless, $86 \%$ of patients in the short TTS group were prescribed antibacterial therapy for a median duration of 4 days despite the absence of bacterial co-infection. This finding underscores the need for continued antimicrobial stewardship to minimize indiscriminant antibacterial use in hospitalized COVID-19 patients. As expected, those with a short TTS had a less complicated course with less than one week length of hospital stay in this cohort. While we identified distinguishing clinical features between groups with varying time to achieve clinical stability, our study is limited by the relatively small number of patients at a single center and the lack of viral load or host immune response measurements to provide a biological basis to our clinical observations.

\section{Conclusion}

Health system resources may be triaged accordingly based on clinical features associated with prompt recovery in patients hospitalized for COVID-19. Concurrent antibacterial therapy needs to be judiciously prescribed to minimize the selection of antimicrobial resistance and untoward adverse effects (e.g. C. difficile diarrhea).

\section{REFERENCES}

1. Phelan AL, Katz R, Gostin LO. The novel coronavirus originating in Wuhan, China: challenges for global health governance. JAMA. 2020; 323 (8):709-710.

2. Gorbalenya AE, Baker SC, Baric RS, et al. Severe acute respiratory syndrome-related coronavirus: the species and its viruses - a statement of the Coronavirus Study Group. bioRxiv. 2020; published online Feb 11. DOI:10.1101/2020.02.07.937862 (preprint).

3. Johns Hopkins University and Medicine Coronavirus Resource Center. Coronavirus COVID-19 Global Cases by the Center for Systems Science and Engineering (CSSE) at Johns Hopkins University (JHU). https://coronavirus.jhu.edu/map.html (accessed November 30, 2020).

4. Harris PA, Taylor R, Thielke R, et al. Research electronic data capture (REDCap): a metadata-driven methodology and workflow process for providing translational research informatics support. J Biomed Inform. 2009;42:377-81.

5. Singer M, Deutschman CS, Seymour CW, et al. The Third International Consensus Definitions of Sepsis and Septic Shock (Sepsis-3). JAMA. 2016;315(8):801-810. 
6. Jin J, Bai P, He W, et al. Gender differences in patients with COVID-19: focus on severity and mortality. Front Public Health. 2020;8:152.

7. Gebhard C, Regitz-Zagrosek V, Neuhauser HK, et al. Impact of sex and gender on COVID-19 outcomes in Europe. Biol Sex Differ. 2020 May 25;11(1):29.

Table 1. Characteristics and Outcomes of Patients Surviving Hospitalization with COVID-19 CHARACTERISTICS

\section{All Patients}

$\mathrm{N}=401$

Short TTS

([?] 3 days)

$\mathrm{N}=174$

Intermediate TTS

(4-10 days)

$\mathrm{N}=135$

Prolonged TTS

(>10 days)

$\mathrm{N}=92$

$p$-value

Demographics

Female

$180(45)$

$89(51)$

$57(42)$

$34(37)$

0.0644

Age, median (IQR), y

64 (50-77)

64 (49-78)

66 (51-77)

$64(50-73)$

0.5982

Residence Prior to Admission

0.0300

Home

291 (73) 
$135(78)$

$93(69)$

$63(68)$

Skilled nursing facility

$102(25)$

37 (21)

$41(30)$

$24(26)$

Outside Hospital/Other

8 (2)

$2(1)$

$1(0.7)$

$5(5)$

Current/Former Smoker

$52(13)$

$21(12)$

$21(16)$

$10(11)$

0.5261

Top 5 Comorbidities

Hypertension

$213(53)$

$86(49)$

$74(55)$

$53(58)$

0.3958

Diabetes

$116(29)$

$46(26)$

$42(31)$

28 (30)

0.6251

Hyperlipidemia/Dyslipidemia

$107(27)$ 
$46(26)$

39 (29)

$22(24)$

0.7040

Dementia

71 (18)

$26(15)$

$30(22)$

15 (16)

0.2316

Chronic Kidney Disease

44 (11)

$15(9)$

$16(12)$

13 (14)

0.3622

None

76 (19)

39 (22)

$23(17)$

$14(15)$

0.2843

Time from Symptom Onset to Hospitalization, median (IQR), days

$4(1-7)$

5 (2-10)

$3(1-7)$

$5(2-7)$

0.0114

Top 3 COVID-19 Symptoms

Fever

$217(54)$

$81(47)$

$78(58)$

58 (63) 
0.0214

Dyspnea

$222(55)$

$87(50)$

$78(58)$

$57(62)$

0.1379

Non-productive cough

$205(51)$

$88(51)$

$64(47)$

$53(58)$

0.3142

Disease Severity upon Admission

Septic shock

$20(5)$

$0(0)$

$5(4)$

$15(16)$

$<0.0001$

Direct ICU admission

$33(8)$

$0(0)$

$10(7)$

$23(25)$

$<0.0001$

Required Supplemental $\mathrm{O}_{2}$

$265(66)$

$95(55)$

99 (73)

$71(77)$

$<0.0001$

Mechanical ventilation

$18(4)$ 
$0(0)$

$3(2)$

$15(16)$

$<0.0001$

Presumed or Culture-Positive Co/Secondary Bacterial Infection ${ }^{-}=$

$138(34)$

$36(21)$

$50(37)$

$52(57)$

$<0.0001$

Receipt of antibacterial therapy

$370(92)$

$149(86)$

$129(96)$

$92(100)$

$<0.0001$

Duration of antibacterial therapy

7 (4-11)

$4(2-6)$

7 (6-10)

$15(11-20)$

$<0.0001$

\section{OUTCOMES}

Developed AKI

$72(18)$

18 (10)

$23(17)$

$31(34)$

$<0.0001$

Developed ARDS

$18(4)$

$0(0)$

$2(1)$

$16(17)$ 
$<0.0001$

Hospital LOS, median (IQR), days

9 (5-15)

$5(3-7)$

9 (8-13)

22 (16-29)

$<0.0001$

${ }^{1}$ Data are presented as number (percentage) of patients unless otherwise indicated.

- = Presumed bacterial co-infection was defined as a procalcitonin level [?] $0.25 \mathrm{ng} / \mathrm{mL}$ upon admission.

Abbreviations: AKI, acute kidney injury; ARDS, acute respiratory distress syndrome; LOS, length of stay

Table 2. Predictors of Prompt Recovery by Multivariable Logistic Regression ${ }^{1}$

\begin{tabular}{lll}
\hline VARIABLES & OR (95\% CI) & $p$-value \\
\hline Female & $1.76(1.13-2.74)$ & 0.01 \\
Time from symptom onset to hospitalization & $1.08(1.03-1.13)$ & 0.001 \\
No Fevers upon admission & $1.64(1.06-2.57)$ & 0.03 \\
No supplemental $\mathrm{O}_{2}$ upon admission & $1.95(1.23-3.07)$ & 0.004 \\
No direct ICU admission & $41.73(2.35-740.35)$ & 0.01 \\
No presumed or culture-positive co/secondary bacterial infection & $2.46(1.51-4.01)$ & 0.0003 \\
\hline
\end{tabular}

The model dependent variable is prompt recovery (short TTS vs. combined intermediate and prolonged TTS cohorts). 